\title{
Evaluation of Conservative Therapy for Intermittent Claudication
}

\author{
Hisao Masaki, MD, PhD; Kazuo Tanemoto, MD, PhD
}

A s described in TASC II, ${ }^{1}$ the Trans-Atlantic Inter-Society Consensus Guidelines, a therapeutic policy for intermittent claudication usually comprises initial treatment by surveillance walking therapy and/or drug therapy for 3-6 months. If no improvement is obtained, endovascular therapy or surgical revascularization is performed. It is stated, however, that endovascular therapy may be performed for proximal iliac artery lesions in cases where the procedure is easily performable. The spontaneous course of intermittent claudication for 5 years reveals stable claudication in 70-80\% of the subjects, with only 5-10\% experiencing severely ischemic extremities. For this reason, patients with this condition are treated mainly by surveillance walking therapy and/or drug therapy. ${ }^{2}$ According to Soga et $a l,{ }^{3}$ the 5 -year patency rate with endovascular therapy for iliac artery lesions was $85.2 \%$. Hence, endovascular therapy has recently been applied positively, producing generally good results for the treatment of iliac artery lesions.

\section{Article p 1486}

We have reported the therapeutic outcomes from exercise therapy and drug therapy performed on patients with intermit- tent claudication at our department. ${ }^{4}$ The study population comprised 61 patients. After being kept at rest in the decubitus position for $10 \mathrm{~min}$, each subject had his or her bilateral ankle and brachial pressures measured simultaneously using the form PWV/ABI ${ }^{\circledR}$ blood pressure pulse wave recorder (Colin Medical Technology Corporation). Subsequently, they underwent a treadmill walking load $(12 \%$ inclination, $2.4 \mathrm{~km} / \mathrm{h}$ speed, 3 min duration). Starting just after completion of the walking, measurements were taken every 2 min until the ankle brachial pressure index (ABPI; ankle systolic pressure/brachial systolic pressure) returned to baseline. If the subject became unable to walk because of pain midway, the walking was discontinued at that time and measurements were taken. Three parameters were measured: (1) resting ABPI, (2) ABPI just after completion of walking, and (3) recovery time (RT) to restoration of baseline ABPI. Patients with ischemic heart disease were excluded.

As a result, the resting ABPI was $0.71 \pm 0.08$ for patients achieving an improvement with conservative treatment and $0.56 \pm 0.11$ for those achieving no improvement; a significantly higher value was obtained for the patients with an improvement. The ABPI for the patients achieving an improvement with conservative treatment was $0.72 \pm 0.07$ before the

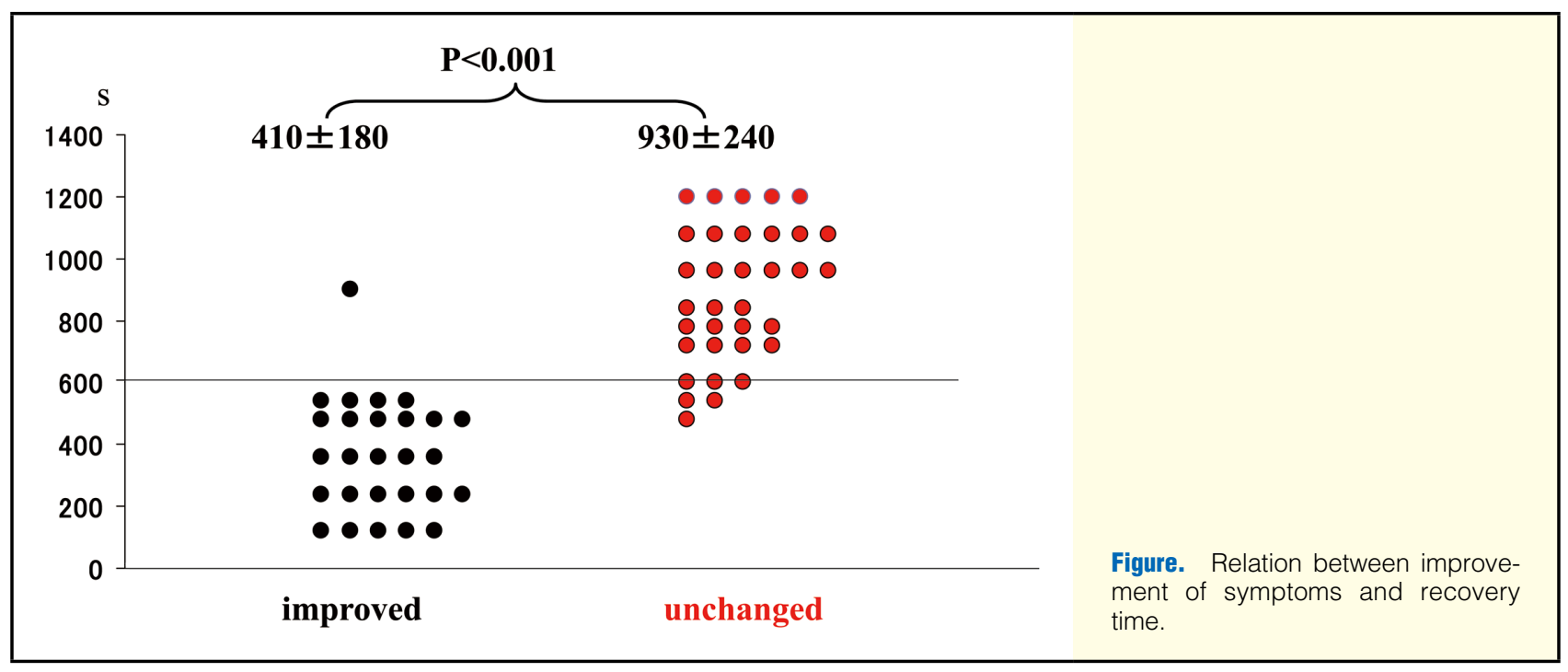

The opinions expressed in this article are not necessarily those of the editors or of the Japanese Circulation Society.

Received March 13, 2012; accepted March 13, 2012; released online April 11, 2012

Division of Cardiovascular Surgery, Department of Surgery, Kawasaki Medical School, Kurashiki, Japan

Mailing address: Hisao Masaki, MD, PhD, Division of Cardiovascular Surgery, Kawasaki Medical School, 577 Matsushima, Kurashiki

701-0192, Japan. E-mail: masaki@med.kawasaki-m.ac.jp

ISSN-1346-9843 doi:10.1253/circj.CJ-12-0333

All rights are reserved to the Japanese Circulation Society. For permissions, please e-mail: cj@j-circ.or.jp 
treatment and $0.74 \pm 0.08$ after 3 months, the difference being statistically insignificant (NS). The ABPI for the patients achieving no improvement was $0.55 \pm 0.10$ before the treatment and $0.53 \pm 0.09$ after 3 months, the difference being statistically insignificant. The RT was $380 \pm 170$ s for the patients achieving an improvement with conservative treatment and $730 \pm 200$ s for the patients achieving no improvement; the latter group had a significantly higher RT $(\mathrm{P}<0.001)$. With a cutoff of $600 \mathrm{~s}$, all patients achieving an improvement had a RT of less than $600 \mathrm{~s}$; all but 3 patients without an improvement had a RT of $\geq 600$ s. Hence, it was concluded that when the RT was shorter than $10 \mathrm{~min}$, conservative treatment was useful, but that when the RT was $\geq 10 \mathrm{~min}$, endovascular therapy or surgical revascularization is preferable (Figure). Ohta et $\mathrm{al}^{5}$ reported on $40-\mathrm{m}$ treadmill walking using an ultrasonic Doppler flowmeter that surveillance walking therapy was effective with RTs of not longer than 13 min but ineffective with longer RTs.

In the group achieving an improvement with exercise therapy, the ABPI remained unchanged after treatment. More specifically, the post-exercise ankle blood pressure and blood flow rate did not increase after treatment. Therefore, the amelioration is attributable not to development of collateral circulation, but to the contribution of elevation of intramuscular metabolic capacity and vascular endothelial functional improvements, as stated in TASC and other reports.

In this issue of the Journal, Serizawa et $\mathrm{al}^{6}$ report that intermittent claudication improved with a new therapy known as extracorporeal shockwave therapy. This treatment is said to promote angiogenesis by acting on VEGF and eNOS. Other reports of swine experiments and human or rabbit studies demonstrate amelioration of myocardial ischemia. Because of the absence of a significant change in ABPI, however, the amelioration is unlikely to be a result of the development of collateral circulation.

Nevertheless, the study is considered valuable because the indexes measured by the authors to evaluate intermittent claudication included maximum walking distance and RT after walking for a given length of time, both of which we make it a rule to measure, and they also included WIQ measurements, although the study population was small. In particular, the maximum walking distance began to extend significantly at week 4, and near-infrared spectroscopy (NIRS) revealed significantly shortened RTs starting at week 8 . When evaluating intermittent claudication using NIRS, convergence time for the Oxy-Hb-Deoxy-Hb dissociation curve serves as a good index, having nearly the same meaning as RT. The tissue oxygenation index (TOI), used in this study, is considered to be a similar index.

Ichiki et $\mathrm{al}^{7}$ reported on RT in NIRS: if the RT is $\leq 180 \mathrm{~s}$, exercise therapy should be performed; if the RT is $180-300$ s, exercise therapy should be performed and, if the condition improves, exercise therapy should be continued, and if the condition does not improve, revascularization surgery should be performed; if the RT $>300 \mathrm{~s}$, revascularization should be performed. Because the study in the current issue of the Journal does not permit extensive analysis because of the small sample size, it will be necessary to increase the study population and analyze RT data in terms of TOI in order to identify patients for whom the treatment is effective.

This treatment is highly expected to be a new option for conservative treatment of intermittent claudication.

\section{References}

1. Norgren I, Hiatt WR, Dormandy JA, Nehler MR, Harris KA, Fowkes FG; TASC II Working Group. Inter-society Consensus for the Management of Peripheral Arterial Disease (TASC II). J Vasc Surg 2007; 45(Suppl S): S5-S67.

2. Matsuo H, Shigematsu H. Patient-based outcomes using the walking impairment questionnaire for patients with peripheral arterial occlusive disease treated with lipo-PGE1. Circ J 2010; 74: 365-370.

3. Soga Y, Yokoi H, Urakawa, Tosaka A, Iwabuchi M, Nobuyoshi M. Long-term clinical outcome after endovascular treatment in patients with intermittent claudication due to iliofemoral artery disease. Circ J 2010; 74: 1689-1695

4. Masaki H, Tabuchi A, Yunoki Y, Kubo H, Hamanaka S, Inagaki E, et al. Therapeutic strategy for intermittent claudication by form PWV/ABI ${ }^{\circledR}$. J Jpn Coll Angiol 2006; 46: 543-547.

5. Ohta T, Sugumoto I, Hida K, Kato M, Takeuchi N, Hosaka M, et al. The prediction of the effects of exercise training in patients with intermittent claudication. Jpn J Vasc Surg 1998; 7: 455-460.

6. Serizawa F, Ito K, Kawamura K, Tsuchida K, Hamada Y, Zukeran T, et al. Extracorporeal shock wave therapy improves the walking ability of patients with peripheral artery disease and intermittent claudication. Circ J 2012; 76: 1486-1493.

7. Ichiki M, Ouchi H, Sai K, Ohnuki K. Evaluation and selection of treatment of intermittent claudication by near-infrared spectrophotometry. Jpn J Vasc Surg 1998; 7: 485-490. 